\section{Impacts on Policy and Decision-Making} Report from the Second Interna-
tional Seville Seminar on Future-
Oriented Technology Analysis

Sevilla, Spanien, 28. - 29. September 2006

\section{Bericht von Michael Rader, ITAS}

\section{Rahmen}

Wie bereits im Titel angedeutet, handelte es sich um ein zweites Treffen zum internationalen Austausch über „Future-Oriented Technology Analysis“ (FTA). Mit dieser Bezeichnung wird versucht, eine gemeinsame Plattform für diverse zukunftsgerichtete Aktivitäten im Zusammenhang mit Technik (wie etwa Technology Foresight und Technology Assessment) zu schaffen. Konzentrierte sich das erste Seminar im Jahre 2004 auf den europäischen / USamerikanischen Erfahrungsaustausch ${ }^{1}$, sprach das zweite eine weltweite Teilnehmerschaft an. So gab es Beiträge vor allem auch aus dem asiatisch-pazifischen Raum und Südamerika. Die Teilnehmerzahl war auf 150 beschränkt, so dass weitere Anmeldungen nicht berücksichtigt werden konnten.

Organisiert wurde das Seminar als Projekt des „European Techno-Economic Policy Support Networks" (ETEPS) ${ }^{2}$ im Auftrag des Institutes for Prospective Technological Studies (IPTS) der Generaldirektion Gemeinsame Forschungsstelle der EU Kommission, das zugleich aktiv an der Vorbereitung mitgewirkt hat ${ }^{3}$. Mit der Vor- und Nachbereitung des Seminars wurde ein internationales „Technical Committee“ betraut, dem in erster Linie Vertreter des ETEPS-Netzwerks ${ }^{4}$ sowie auch eine Reihe von international renommierten Experten ${ }^{5}$ angehörten. Das Technical Committee bereitete das Seminar unter anderem durch die Erstellung einer Anzahl von „anchor papers“ vor, die den Autoren der eingereichten Beiträge Hinweise auf die angestrebte Stoßrichtung des Seminars geben sollten.

Beschäftigte sich das 2004er Seminar noch mit „Perspektiven und Herausforderungen“, so strebte die Neuauflage den Austausch über die Auswirkungen auf Politik und Entscheidungsfindung an.

Wie stark das Interesse an dem Thema war, zeigte die hohe Anzahl von eingereichten Beiträgen, von denen das Technical Committee etwa 50 Vorträge und 17 „e-Poster“ für das Seminar annahm. Letztere sollten insbesondere zur Information über noch nicht abgeschlossene Projekte dienen und waren Gegenstand eines „Wettbewerbs“. In diesem Wettbewerb sollte es auch um die Darbietung wissenschaftlicher Inhalte mit den Möglichkeiten von Multimedia gehen. Letzten Endes waren die Beiträge aber ausschließlich ziemlich konventionelle Powerpoint-Präsentationen, zum Teil von deutlich größerer Länge als die Vorträge im Plenum oder den Sektionen. Als unglücklich hat sich der Rahmen für ihre Präsentationen erwiesen: als programmierte Sequenz in den Kaffee- und Mittagspausen und beschränkt auf einen kleinen versteckten Raum im IPTS-Gebäude. ${ }^{6}$

\section{Ablauf}

Die Veranstaltung wurde mit Vorträgen von Theodore Gordon ("Making better decisions in an uncertain world“), Gülsün Saglamer (,The future role of University for a creative and innovative society“), Eleonora Barbieri Masini („Futures, technologies and public awareness“") ${ }^{7}$ als Repräsentanten aus Wissenschaft und Forschung, und Dietmar Theis („Developing scenarios for future technologies and food for thought about future lifeworlds“) als Repräsentant der global tätigen europäischen Industrie eingeleitet.

Die 30minütigen Vorträge der Konferenzteilnehmer wurden folgenden fünf Themen zugeordnet, zu denen es jeweils ein ,anchor paper“ gegeben hatte und die Gegenstand von Parallelsitzungen während des ersten Teils des Seminars waren:

1. FTA Assumptions, Methods and Approaches (z. B. zum European Foresight Monitoring Network EFMN);

2. FTA Evaluation, Impact and Learning (z. B. das Lernen von Science \& Technology Studies für den Einsatz als Instrument der Politikplanung); 
3. FTA in the Business Context (z. B. eine zukunftsorientierte Bestandaufnahme für Europa);

4. FTA in Higher Education (also der Einsatz von FTA als Planungsinstrument für Universitäten);

5. FTA in Developing Countries (Beispiele waren Sri Lanka, Brasilien, Kolumbien).

Die zweite Hälfte der Veranstaltung bestand aus der Arbeit, die von Mitgliedern des Technical Committees moderiert wurden. Diese Kleingruppen beschäftigten sich mit sechs verschiedenen Aspekten der Governance von FTA:

- Building Capacity,

- Customer-Client Relations,

- Quality and Ethical Standards,

- Preparedness to address Global Problems,

- Building Links across the FTA Community,

- Evaluation and Monitoring.

Jede Arbeitsgruppe hatte die Aufgabe, drei Maßnahmen vorzuschlagen, die nach Meinung der Teilnehmer für den behandelten Aspekt der FTA Governance am vordringlichsten waren. Der Autor leitete die Arbeitsgruppe zu ,building capacity“, die folgende drei Maßnahmen für vordringlich hielt:

- Einrichtung eines Wikipedia ${ }^{8}$ („Wiki“) für FTA,

- Erstellung eines Handbuchs zu FTA,

- Eröffnung eines Internetportals für FTA.

\section{Eindrücke und Auswirkungen}

Da bis auf einen alle Vorträge auf der SeminarWebseite enthalten sind, beschränkt sich dieser Bericht in der Folge auf wenige Impressionen und die Folgeaktivitäten, die sich aus der Nachbereitung des Seminars ergeben haben:

1. Nach wie vor ist unklar, was „Future Oriented Technology Analysis“ genau sein soll, insbesondere, ob und wie sie sich von „Foresight“ abhebt. Häufig wird in Beiträgen zunächst das Kürzel „FTA“ verwendet, um in der Folgezeit von „foresight“ zu sprechen. So wurde dann die geringe Repräsentanz von Technology Assessment auch von einem der Hauptorganisatoren beklagt (in informellem Rahmen), doch bleibt festzuhalten, dass manche Foresight-Aktivitäten dem sehr nahe kommen, was zu anderer
Zeit und andernorts als „Technology Assessment“ bezeichnet wird. Das ,anchor paper“" von Alan Porter und dem Autor dieses Konferenzberichts über „FTA Assumptions: Methods and Approaches“ thematisiert unter anderem diesen Aspekt. ${ }^{9}$

2. Nach wie vor ist es sehr schwierig, die Impacts von FTA-Studien in den Griff zu bekommen und die allerwenigsten Beiträge konnten Konkretes dazu aussagen. Der Vorschlag des TAMI-Projekts ${ }^{10}$, eine Mehrzahl von Zielen für jedes Projekt zu definieren, um auf diese Weise Impacts leichter nachweisen zu können, scheint hier noch keine Resonanz gefunden zu haben, wird aber in der revidierten Fassung für eine Buchpublikation des Porter / Rader-Papiers angesprochen.

3. Unklar ist, ob Technik bzw. Technologie eine essentielle Komponente von Foresight ist, d. h. ob sich Zukunftsstudien auf jeden Fall auch mit Technologie befassen müssen. Auf jeden Fall hat sich Foresight / Technologie-Vorausschau in jenen Regionen, in denen sie ursprünglich einen technologischen Hintergrund hatte, auf weitere Fragen wie der Entwicklung der Gesellschaft und der Problematik des gesellschaftlichen Kontextes der Entwicklung und Anwendung der Technik ausgedehnt.

4. Ausgewählte Beiträge werden in einem Tagungsband bzw. Sonderausgaben von zwei international renommierten Zeitschriften („Technological Forecasting and Social Change" und "Technology Analysis and Strategic Management”) erscheinen. Diese werden von Mitgliedern des Technical Committees betreut.

5. Die Anregungen aus den Governance Sessions werden vom Technical Committee aufgegriffen und auch praktisch umgesetzt z. B. die Schaffung eines TFA-,,Portals“ auf Grundlage der schon bestehenden Portale zu Foresight oder die Einrichtung von MailingListen zu verschiedenen Themen wie:

- Building Capacity (e.g. through training),

- Customer Client Relations / Quality,

- Evaluation and Monitoring,

- Preparedness to Address Global Problems,

- Building Community Links (e.g. towards a professional association?). 
Eine weitere Anregung betraf die Erstellung eines "Handbuchs zur TFA" auf Basis eines noch einzurichtenden Wiki (s. o.). Eine erste Aufgabe in diesem Bereich wird sicher nochmals das Abstecken des Feldes und die Identifikation der FTA Community sein.

Da bei der EU-Kommission ein grundlegendes Revirement zu Beginn des siebten Rahmenprogramms stattfindet und Foresight kein eigenständiges Referat bekommt, kommt dem IPTS bei diesen Aktivitäten eine herausragende Rolle zu. Einigermaßen fest steht, dass es ein drittes Seminar in Sevilla zu „Future-Oriented Technology Analysis” geben wird. Auch über weitere Entwicklungen wird in dieser Zeitschrift informiert werden.

\section{Literatur}

Decker, M.; Ladikas, M. (Hg.), 2004: Bridges between science, society and policy. Technology assessment - Methods and impacts. Berlin: Springer Verlag

\section{Anmerkungen}

1) Ein Bericht hierzu wurde in TATuP 13/2 (2004) veröffentlicht und steht auf dem ITAS-Server unter http://www.itas.fzk.de/tatup/042/fied04a. htm zur Verfügung (Anm. d. Red.).

2) Zu ETEPS siehe den Bericht in der Rubrik TAInstitutionen in diesem Heft.

3) Für ETEPS waren dies die Foresight-Experten von PREST aus Großbritannien, Luke Georghiou und Mike Keenan, für das IPTS Fabiana Scapolo, Ron Johnston und Cristiano Cagnin der Foresight-Gruppe.

4) Kerstin Cuhls (FHG-ISI) Jennifer CassingenaHarper (Malta Council for Science and Technology) Michael Rader (FZK-ITAS).

5) Alan Porter (Georgia Institute of Technology), Mario Albornoz (Centro de Estudios sobre Ciencia, Desarrollo y Educación Superior, Buenos Aires) sowie Remi Barré (Conservatoire National des Arts et Métiers, Paris).

6) Sie sind jedoch ebenso wie die Vorträge auf der Konferenz-Webseite (http://forera.jrc.es/fta/intro. html) vertreten.

7) Eleonora Barbieri Masini ist emeritierte Professorin der Gregorianischen Universität Rom und eine „Grande Dame“ der Zukunftsforschung.

8) Nach dem Vorbild der Internetenzyklopädie Wikipedia kollektiv erstellte Artikelsammlung.
9) Verfügbar auf der Konferenz-Webseite unter: http://forera.jrc.es/documents/papers/anchor/FT A-Paper\%201-Porter\%20\%20RaderFinalPaper V4-aug5.pdf

10) Siehe Decker, Ladikas 2004. Ein Bericht über die Ergebnisse des TAMI-Projekts wurde in TATuP 13/1 (2004) veröffentlicht und steht auf dem ITAS-Server unter http://www.itas.fzk.de/ tatup/041/dela04a.htm (Anm. d. Red.).

\section{First WORKS conference The Transformation of Work in a Global Knowledge Economy: Towards a Conceptual Frame- work}

Chania, Greece, September 21 - 22, 2006

\section{By Martin Bechmann, Linda Nierling, ITAS and Tobias Woll, Universidade Nova de Lisboa, Portugal}

\section{Project background and conference aims}

In the last decade, "Globalisation" has become a common topic of public debate. Like a force of nature, it seems to be unlimited in scope and consequences and has been taken as an argument to sanctify the restructuring of firms as well as of nation states and welfare systems. Production in different sectors of industry is increasingly organised in value chains which extend over national boundaries, act in varying environments and are becoming more flexible due to modularisation and segmentation into autonomous and partially autonomous business functions. Undoubtedly, globalisation has a wide variety of effects on societies. Nevertheless, detailed and systematic expertise on how the global integration of markets and value chains impacts companies, working life and political and legislative options, are still rare. For the social sciences, this poses new challenges in terms of describing and conceptualising globalisation, in bridging the gap between analysis on the micro- and macro-levels and in terms of methodology. The examination of value chains of increasing complexity also re- 